\title{
Potential Role of Arsenic Resistant Bacteria in Bioremediation: Current Status and Future Prospects
}

\author{
Ghanshyam Kumar Satyapal, Shikha Rani, Mukund Kumar and Nitish Kumar* \\ Centre for Biological Sciences (Biotechnology), School of Earth, Biological and Environmental Sciences, Central University of South Bihar, Patna, Bihar, India
}

\begin{abstract}
Arsenic is known as a toxic metalloid, which primarily exists in inorganic form (AsIll and AsV). The industrialization and anthropogenic activities are the source of arsenic in the environment. Some microorganisms under heavy metal stress, developed resistance against them and evolved various strategies to resist against the metal stress. Detoxification of arsenic includes uptake of $\mathrm{AsV}$ in the form of phosphate by phosphate transporters, uptake of AsIII in the form of arsenite by aquaglyceroporins, reduction of AsV to AsIII by arsenate reductase, oxidation and methylation of AsIII by arsenate oxidase and methyltransferase respectively and finally extrusion or sequestration of AsIII. Many bacteria have been reported having redox potential for arsenic. Some genetically modified or engineered bacteria, C. Glutamicum, have been developed which show increased efficiency for arsenic transformation and could be used as biocontainers for bioaccumulation of arsenic.
\end{abstract}

Keywords: Arsenic; Arsenate; Arsenite; Uptake; Extrusion; Bioremediation; Bioaccumulation

\section{Introduction}

The metalloid arsenic (As) is a member of group $\mathrm{V}$ of the periodic table of elements and is thus classified as a heavy metal. Arsenic occurs in nature in four oxidation states $(+5,+3,0$, and -3$)$, with pentavalent arsenate $[+5, \mathrm{As}(\mathrm{V})]$, and trivalent arsenite $[+3, \mathrm{As}(\mathrm{III})]$ being the most common forms. Under anoxic conditions, arsenite can be reduced by microorganisms in soil to the volatile compounds arsine (AsH3) and methylarsines, these compounds being the most toxic forms of arsenic [1]. There is a wide distribution of arsenic in the environment due to both natural and anthropogenic activities and it is often found in food, soil and air borne particles [2]. The mining and smelting activities results in the release of arsenic into the environment in inorganic forms [As(III) and As(V)] [3]. The main sources of exposure happen to be drinking water and food. Great concentrations are deposited in the liver, kidney, lungs and skin. Besides these, small concentrations could be found in the bone and muscles while accumulation in hair and nails is observed as a result of chronic exposure. Proposed mechanisms of action in exerting these effects include mitochondrial damage, altered DNA repair, altered DNA methylation, oxidative stress, cell proliferation, co-carcinogenesis and tumour promotion [2]. The ubiquity of arsenic leads to develop various strategies for growing in metal stressed environment. Bioremediation is the process of mitigating environmental pollutants using microorganisms. Bioremediation of different forms of arsenic by microbial community involves their oxidation, reduction, methylation and intracellular bioaccumulation. Arsenate uptake is mediated by phosphate transporter proteins Pst and Pit whereas arsenite is uptaken by glycerol transporter GlpF [4]. The As(III) is pumped out through the by the ArsAB-ATPase [5]. In certain condition metalloid tolerance contributed by aquaglyceroporins since they are bidirectional channels that transport substrates down the concentration gradient. Thus theoretically aquaglyceroporins could be involved not only in uptake of metalloid but also in their efflux which contributes to metalloid tolerance [6]. The occurrence of ars operons in the genomes and plasmids provides arsenate reduction ability to the bacteria, e.g. arsenate reductase (ArsC), ArsA and ArsB to be a component of ArsAB-ATPase efflux pump [3]. Similarly, the aox operons provide arsenite-oxidising ability to the bacteria. ArsM is a methyltransferase involve in enzymatic arsenic methylation by using
S-adenosylmethionine (SAM) as a methyl source [4,7]. The arsenic reducing bacteria of genus Pseudomonas, VIbrio, Enterobacter and Bacillus have been isolated and they are tolerant upto $200 \mathrm{mM}$ of arsenate [8]. NT-26 has been reported as the fastest arsenite oxidiser [9]. B1-CDA strain can accumulate arsenic inside the cells [10]. A mutant C. glutamicum strain is engineered for accumulation of the arsenic intracellularly and used as a biocontainers [11].

\section{Arsenic Uptake and Extrusion by Bacteria}

Arsenic is ubiquitous in the environment. The molecular similarity of arsenic to the substrates of various membrane transporter proteins in prokaryotes permeates their uptake. In aqueous solution at physiological $\mathrm{pH}$ AsIII, exits as $\mathrm{As}(\mathrm{OH})_{3}$, which is structurally similar to glycerol. Whereas, AsV acts as a structural analog of phosphate and is taken up by the membrane phosphate transporters [10]. GlpF is an aquaglyceroprotein which involves in transport of AsIII across the cell membrane. Homologies of GlpF have been found in several bacteria. Pit and Pst are the phosphate transporter proteins which facilitate the uptake of AsV [7]. Pst is specific transport system for phosphate whereas Pit is general transport mechanism. The structural similarity of arsenate with phosphate leads to its easily uptake through Pit transport system [12]. Since, Pst transport AsV less efficiently hence the microbial community which are exposed to high level of arsenate only express Pst to reduce the AsV uptake [7]. In bacteria, arsenic extrusion is done by two mechanisms. One is carrier-mediated efflux via an arsenite carrier protein, where energy is supplied by the membrane potential of the cell, and the other by an arsenite-translocating ATPase [4,11]. The bacteria having three gene operon $\operatorname{ars} R B C$ can extrude arsenite by ArsB alone whereas the five gene operon, arsRDABC, can extrude arsenite by the

*Corresponding author: Nitish Kumar, Centre for Biological Sciences (Biotechnology) School of Earth, Biological and Environmental Sciences, Central University of South Bihar, Patna, Bihar, India, Tel: 0612222 6535; E-mail: nitish@cub.ac.in

Received May 11, 2016; Accepted June 09, 2016; Published June 16, 2016

Citation: Satyapal GK, Rani S, Kumar M, Kumar N (2016) Potential Role of Arsenic Resistant Bacteria in Bioremediation: Current Status and Future Prospects. J Microb Biochem Technol 8: 256-258. doi: 10.4172/1948-5948.1000294

Copyright: () 2016 Satyapal GK, et al. This is an open-access article distributed under the terms of the Creative Commons Attribution License, which permits unrestricted use, distribution, and reproduction in any medium, provided the original author and source are credited. 
ArsAB pump. An ArsAB complex from when ArsA is co-expressed with ArsB which is obligatory coupled to ATP. E. coli synthesizing ArsA from plasmid R773 arsRDABC operon are more resistant to arsenite because the ArsAB ATPaes can extrude arsenite more efficiently than ArsB alone [4].

\section{Genes Involved in Arsenic Transformation}

Arsenic tolerance in bacteria is mediated by the gene products of the ars operon. In $E$. coli genome ars $R B C$ a three gene operon is present whereas in $S$. aureus arsRDABC a five gene operon is present [4]. The $\operatorname{ars} C$ encodes for an arsenate reductase, $\operatorname{ars} B$ and $\operatorname{ars} A$ encodes for an arsenite efflux pump, i.e., ArsAB ATPase, and arsR encodes for a transcriptional regulator [13]. The arsD acts as arsenic chaperone that transfers arsenite from the glutathione-bound complexes to ArsA subunit of the ArsAB complex and activates it. Both operons may be present in one strain, as e.g. in T. arsenitoxidans 3As. The arr operon including the $\operatorname{arr} A$ and $\operatorname{arr} B$ genes is involved in arsenate reduction in the periplasm of the bacteria. The product of ars $M$ gene is involved in the methylation of arsenite [7]. The four contiguous genes encoding arsenite oxidase from Centibacterium arsenoxidans, and named as aox $A B C D$. In $A$. faecalis, aso $A$ gene product is homologous to the aox $B$ gene product; similarly $a s o B$ gene product is identical to the aox $A$ gene product [14]. In H. arsenoxidans, aoxS gene product is a sensor kinase and aoxR gene product acts as regulator for the aox operon, both are involve in quorum sensing [15].

\section{Arsenite Oxidation in Bacteria}

Bacteria can oxidise AsIII into a less toxic form of arsenic, i.e., AsV. The arsenite oxidase catalyses the arsenite oxidation [7]. The arsenite oxidation is done in the periplasm of the bacteria. AoxS is a sensor kinase which senses the presence of arsenite and activates AoxR, a regulator protein. This AoxR control the expression of the aox operon in association with the RpoN (an alternative $\sigma^{54}$ of RNA polymerase). This RpoN is essential for the initiation of the arsenite oxidase transcription in A. tumefaciens. In H. arsenoxidans DnaJ, is a heat shock protein, is required for the proper folding of the AoxR regulators [15]. DnaJ is known to be essential for the expression of the flhDC flagellar master operon in Escherichia coli which is involve in bacterial motility [15]. Aox $\mathrm{AB}$ complex, arsenite oxidase, is synthesized after expression of the aox operon and exported to the periplasm by a Tat (Twin-Arginine Translocation) protein export pathway. Where, in the periplasm, it involves in the oxidation of AsIII into the AsV [14].

\section{Arsenate Reduction in Bacteria}

In prokaryotes, arsenate reduction system is of two types; cytoplasmic arsenate reduction and periplasmin arsenate reduction. When $\mathrm{AsV}$ is taken up by the pst and pit membrane transporters, arsC, an arsenate reductase enzyme involve in the reduction of the AsV to AsIII and leads to its extrusion through the arsAB pump. Whereas ArrA priplasmic respiratory arsenate reductase enzyme [7]. Homologues of the ars $C$ gene present in both; plasmids andthe chromosomes. In cytoplasmic reduction ArsC utilizes glutaredoxins which serves as source of reducing potential. In this reaction cascade, arsenate first binds to the anion site in the $\mathrm{Ars} C$ then it forms a arsenate thioester intermediate with the active site Cys12.It is then reduced in two steps by glutaredoxin and glutathione, producing the Cystic2-S-As(III) intermediate, which hydrolyzes to release arsenite [4]. The reduced AsIII is extruded from the cell or sequestered in the intracellular compartments, either as free arsenite or as conjugates with glutathione or other thiols.

\section{Arsenic Methylation}

Methylation is thought to be a detoxification process, however, less is known for bacterial system. Methylated products are not less toxic than inorganic forms of arsenic [16]. Some compounds of the pathway have been shown to be more toxic to eukaryotic cell lines then the inorganic forms of arsenic. In methylation process, production of trimethyl arsine gas concludes that the increased volatility of methylated arsenicals can increase the toxicity of some intermediates [7]. The methylation of arsenic is enzymatic, requiring $S$-adenosylmethionine (SAM) and a methyltransferase. ArsM, a methyltransferase, encoded by arsM gene methylate arsenite into a monomethyl arsenite (MMAs(III)). This MMAsIII is further methylated to a dimethyl arsenite (DMAs(III)) followed by a trimethyl arsine (TMAs(III)) as s final product. These methyl arsenicals can be transported out the cell by a diffusion process [17]. However, in prokaryotes less known about demethylation mechanisms. Some microorganisms, e.g., Alcligenes, Pseudomonas and Mycobacterium species shown to demethylate mono and dimethyl arsenic compounds [16].

\section{Bacterial Implication in Arsenic Bioremediation}

Recent studies have described the isolation and characterization of arsenite-oxidizing, arsenate-reducing bacteria. Some mutant strains of bacteria were also engineered. The aerobic bacteria were isolated and identified as they belongs to the Acinetobacter, Comamonas, Pseudomonas, Stenotrophomonas, Delftia, Agrobacterium and Bacillus species [18]. NT-26 has been reported as the fastest arsenite oxidiser having doubling time of $7.6 \mathrm{~h}$ [9]. Bacteria are able to anaerobically utilize arsenate as an electron acceptor and can use oxygen as electron donor and arsenite as electron donor in a chemolithotropic manner. Some isolated genera have not been previously reported to have direct interaction with the arsenic species, for examples, Aeromonas and Exiguobacterium. Two strains CA1 and WK6 belonging to the species Aeromonas and Exiguobacterium, respectively. The CA1 indicate a novel pathway for arsenate reduction as there was no $\operatorname{ars} B$ and $\operatorname{ars} C$ was observed. Whereas, strain WK6 shows ars $B$ but not $\operatorname{ars} C$, thus indicates an arsC-independent pathway. Chrysiogenes arsenatis can respire aerobically by using arsenate as a terminal electron acceptor and acetate as electron donor [19]. A new strain MM-7 is the first roprt of AsIII oxidising bacterium from the genus, Stenotrophomonas. It can completely oxidise $500 \mu \mathrm{M}$ of arsenite in $12 \mathrm{~h}$ of incubation at $\mathrm{pH}$ ranging from 5 to 7 [20]. Pseudomonas lubricans showed high resistance against arsenite up to $3 \mathrm{mg} / \mathrm{ml}$ [21]. Non-enzymatic processes such as, adsorption, is characterized by the non-specific binding of metal ions to extracellular/cell surface associated polysaccharides and proteins. Microbial biosorbent may be an active or passive process or exhibit both; it depends upon the microbial species that involved in metal uptake. The passive uptake process is relatively nonspecific to metal species but the active process is comparatively slow method and depends on the cellular metabolism. In active process, specific proteins like metallothionins form complexes with the heavy metals. Both the active and passive processes may occur simultaneously. Microbial species with high cell wall chitin contents acts as an effective biosorbent in addition to the chitosan and glucans. The walls of fungi, yeasts, and algae, are also efficient metal biosorbents. Gram-positive bacteria can attach higher concentrations of metals than that of the Gram-negative bacteria. Bioaccumulation is an energy-dependent heavy metal transport system. The potential of bioaccumulation mechanisms of heavy metal influx across the bacterial membranes include ion pumps, ion channels, carrier mediated transport, endocytosis and lipid permeation [22]. Genetically modified bacteria have been developed as bioreporter for inorganic 
species in the environment. These bioreporters based on the genetic constructs in which an ars $R$ operator and promoter sequence is joined with the sequence of the reporter gene, such as luciferase, $\beta$-galactose, an autofluorescent protein or cytochrome $c$ peroxidise [7]. Among the many possible arsenic bioremediation processes, the intracellular accumulation by bacteria is preferred. The strain B1-CDA cells, when exposed to arsenate, uptake and accumulate different forms of arsenics inside the cells, such as free forms arsenic, meta-arsenite, orthoarsenite and arsenate [10]. A mutant C. glutamicum strain is engineered for accumulation of the arsenic intracellularly. Arsenate accumulation was increased by the removal of the ArsC (arsenate reductase) and by the removal of the Acr3 (efflux) systems. Arsenate accumulation was increased 28-30 folds in the mutant strain than the control strain. AsIII biocontainers were also engineered by removing the AsIII efflux system. Overexpression of GlpF protein in C. glutamicum can results in increased uptake of the arsenite. As engineered C. glutamicum serves as biocontainers by accumulating the large amount of arsenic. It offers two-step detoxification system for arsenic. In the first step, AsIII was oxidized to AsV by Ochrobacterium tritici; followed by a second step, $\mathrm{AsV}$ and remaining AsIII accumulation by engineered C. glutamicum [11].

\section{Conclusion}

In the past few years, a large amount of information has been published on arsenic metabolism, in bacteria, on the genes involved in these processes and their regulation. They improved our understanding of functioning, diversity and the evolution of arsenic-resistant bacteria. Biosorbent, bioaccumulation and genetically engineered biocontainers provides great openings for their use in arsenic bioremediation. These studies give an integrated view of microorganisms present in the arsenic contaminated environments, their role and their relationships.

\section{References}

1. Mateos LM, Ordóñez E, Letek M, Gil JA (2006) Corynebacterium glutamicum as a model bacterium for the bioremediation of arsenic. Int Microbiol 9: 207-215.

2. Obinaju, Ebele B (2009) Mechanisms of arsenic toxicity and carcinogenesis. African Journal of Biochemistry Research 3: 232-237.

3. Lewis AS, Reid KR, Pollock MC, Campleman SL (2012) Speciated arsenic in air: Measurement methodology and risk assessment considerations. J Air Waste Manag Assoc 62: 2-17.

4. Rosen BP (2002) Biochemistry of arsenic detoxification. FEBS Lett 529: 86-92.

5. Mukhopadhyay R, Rosen BP (2002) Arsenate reductases in prokaryotes and eukaryotes. Environ Health Perspect 110 Suppl 5: 745-748.

6. Maciaszczyk-Dziubinska E, Wawrzycka D, Wysocki R (2012) Arsenic and antimony transporters in eukaryotes. Int J Mol Sci 13: 3527-3548.

7. Kruger MC, Bertin PN, Heipieper HJ, Arsène-Ploetze F (2013) Bacterial metabolism of environmental arsenic-mechanisms and biotechnological applications. Appl Microbiol Biotechnol 97:3827-3841.

8. Liao VH, Chu YJ, Su YC, Hsiao SY, Wei CC, et al. (2011) Arsenite-oxidizing and arsenate-reducing bacteria associated with arsenic-rich groundwater in Taiwan. J Contam Hydrol 123: 20-29.
9. Santini JM, Sly LI, Schnagl RD, Macy JM (2000) A new chemolithoautotrophic arsenite-oxidizing bacterium isolated from a gold mine: Phylogenetic physiological and preliminary biochemical Studies. Applied and Environmental Microbiology 66: 92-97.

10. Rahman A, Nahar N, Nawani NN, Jass J, Desale P, et al. (2014) Isolation and characterization of a Lysinibacillus strain B1-CDA showing potential for bioremediation of arsenics from contaminated water. Journal of Environmental Science and Health 49: 1349-1360.

11. Villadangos AF, Ordóñez E, Pedre B, Messens J, Gil JA, et al. (2014) Engineered coryneform bacteria as a bio-tool for arsenic remediation. Appl Microbiol Biotechnol 98: 10143-10152.

12. Bertin PN, Heinrich-Salmeron A, Pelletier E, Goulhen-Chollet F, ArsènePloetze F, et al. (2011) Metabolic diversity among main microorganisms inside an arsenic-rich ecosystem revealed by meta- and proteo-genomics. ISME J 5 : 1735-1747.

13. Arsène-Ploetze F, Koechler S, Marchal M, Coppée JY, Chandler M, et al. (2010) Structure, function and evolution of the Thiomonas spp. genome. PLoS Genet 6: e1000859.

14. Silver S, Phung LT (2005) Genes and enzymes involved in bacterial oxidation and reduction of inorganic arsenic. Appl Environ Microbiol 71: 599-608.

15. Koechler S, Cleiss-Arnold J, Proux C, Sismeiro O, Dillies M, et al. (2010) Multiple controls affect arsenite oxidase gene expression in Herminiimonas arsenicoxydans. BMC Microbiology 10: 53.

16. Stolz JF, Basu P, Santini JM, Oremland RS (2006) Arsenic and selenium in microbial metabolism. Annu Rev Microbiol 60: 107-130.

17. Hughes MF (2002) Arsenic toxicity and potential mechanisms of action. Toxicol Lett 133: 1-16.

18. Cai L, Liu G, Rensing C, Wang G (2009) Genes involved in arsenic transformation and resistance associated with different levels of arseniccontaminated soils. BMC Microbiol 9: 4.

19. Anderson CR, Cook GM (2004) Isolation and characterization of arsenatereducing bacteria from arsenic-contaminated sites in New Zealand. Curr Microbiol 48: 341-347.

20. Bahar MM, Megharaj M, Naidu R (2012) Arsenic bioremediation potential of a new arsenite-oxidizing bacterium Stenotrophomonas sp. MM-7 isolated from soil. Biodegradation 23: 803-812.

21. Rehman A, Butt SA, Hasnain S (2010) Isolation and characterization of arsenite oxidizing Pseudomonas lubricans and its potential use in bioremediation of wastewater. African Journal of Biotechnology 9: 1493-1498.

22. Ahmed M (2012) Implications of bacterial resistance against heavy metals in bioremediation: A Review. IIOABJ 3: 39-46. 